DISCUSSION/SIGNIFICANCE OF IMPACT: Our contributions here are expected to be the elucidation of European ancestry as a risk factor for AF. These contributions will be significant because it can provide a robust scientific basis for larger GWAS studies in the Puerto Rican community and further narrow down the mechanism specific to this population. Research in this subject could lead to early identification of patients with high risk of developing atrial fibrillation and further decrease incidence and disease burden in the PRH population. Puerto Rican Hispanics have an exclusive genetic admixture that makes for an appealing research subject that could deliver unique results.

3571

Expression profile of the ribbon synapse protein Ribeye in the zebrafish

Courtney Frederick, $\mathrm{PhD}^{1}$ and David Zenisek

${ }^{1}$ Yale School of Medicine

OBJECTIVES/SPECIFIC AIMS: Although two Ribeye protein isoforms have been identified in zebrafish, information about the identities of their variants is incomplete. This study aims to identify and characterize both of the Ribeye isoforms and their splice variants. METHODS/STUDY POPULATION: Immunohistochemistry was performed on the retina and neuromasts of zebrafish larva and adults.Ribeye expression was analyzed by western blot. Ribeye proteins will be separated, isolated and identified by mass spectrophotometry. RESULTS/ANTICIPATED RESULTS: Immunohistochemistry performed on larval and adult zebrafish retinas revealed the expression of Ribeye A in the inner and outer plexiform layers. Ribeye B was likewise expressed in both plexiform layers in larval zebrafish, but more pronounced expression in the outer plexiform layer in the adult zebrafish retina. Immunohistochemical experiments also demonstrated the co-expression of both Ribeye isoforms in the hair cells of both larval and adult neuromasts. Analysis of Ribeye expression by western blot showed the presence of more than the three previously identified variants. Current experiments are being conducted to characterize the additional Ribeye variants. We expect to identify the residual Ribeye protein as a result of this analysis. DISCUSSION/ SIGNIFICANCE OF IMPACT: This study is necessary in order to gain a clear understanding of Ribeye expression in zebrafish tissues. Doing so will enable us to target this protein for gene editing to address outstanding questions about the mechanisms that govern ribbon synapse function. Synapse and synapse-associated proteins are involved in a wide-array of diseases that arise as a result of their dysfunction (e.g. blindness, deafness, bradycardia, autism spectrum disorders, and schizophrenia). Thus, it is important for us to identify the shared and distinct mechanisms that give rise to diseases associated with synaptic dysregulation. Such information could provide the basis for novel therapeutic interventions for synaptic disorders.

3322

Functional brain mechanisms of sensorimotor deficits in individuals with autism spectrum disorder

Kathryn Unruh ${ }^{1}$, Laura Martin, Grant Magnon, David Vaillancourt, John Sweeney and Matthew Mosconi

${ }^{1}$ University of Kansas Frontiers

OBJECTIVES/SPECIFIC AIMS: Abnormalities in sensorimotor behavior are present in the majority of individuals with ASD and associated with core symptoms. Cortico-cerebellar networks that control sensorimotor behavior have been implicated in ASD, but little is known about their function during sensorimotor actions. The purpose of this functional magnetic resonance imaging (fMRI) study was to examine cortical-cerebellar function during feedback-guided motor behavior in ASD. METHODS/STUDY POPULATION: Individuals with ASD (11-30 years; $\mathrm{N}=18)$ and age-matched controls $(\mathrm{N}=15)$ completed a visuomotor task of feedback-guided precision gripping during fMRI. Participants pressed with their right thumb and forefinger on a force transducer while viewing a green FORCE bar on a screen that moved upwards with increased force toward a fixed white TARGET bar. Individuals were instructed to maintain the FORCE bar at the level of the TARGET bar for 24 seconds. Target force levels were set at $20 \%$ and $60 \%$ of each participant's maximum voluntary contraction (MVC). Force variability was characterized as the coefficient of variation (i.e., standard deviation of the force time series / mean force output; CoV). RESULTS/ANTICIPATED RESULTS: Mean force did not differ between groups indicating participants were able to follow task demands. Participants with ASD showed increased force variability $(\mathrm{F}(1,30)=5.214, \mathrm{p}=0.03)$ at both $20 \%(\mathrm{~d}=.45)$ and $60 \%(\mathrm{~d}=.77)$ MVC compared to controls. Compared to controls, individuals with ASD showed decreased activation in left angular gyrus during the visuomotor task compared to rest (AG; maximum $\mathrm{t}=4.31$ ). Individuals with ASD also showed greater visuomotor activation compared to controls in ipsilateral ventral M1, extending anteriorly into posterior ventral pre-motor cortex (PMv; maximum $t=-4.06$, cluster size $=38$ voxels). This difference reflected the finding that control participants showed a selective deactivation of ipsilateral $\mathrm{M} 1 / \mathrm{PMv}$ during visuomotor behavior, whereas individuals with ASD did not show this pattern. A significant group $\mathrm{x}$ force interaction was observed for contralateral Crus I activation (maximum $\mathrm{t}=-2.42$ ) that was driven by an increase in activity during $60 \%$ compared to $20 \%$ MVC in control participants, while individuals with ASD showed no change in Crus I activation between force levels. DISCUSSION/SIGNIFICANCE OF IMPACT: Increased force variability in individuals with ASD suggests impaired processing of sensory feedback to guide precision motor behaviors. Individuals with ASD did not show deactivation of right motor cortex during visuomotor behavior relative to rest, suggesting reduced ability to selectively modulate motor cortical output. Reduced activation in left AG may reflect an inability to integrate visual, haptic, and proprioceptive inputs to reactively adjust ongoing motor output. Failure to show force-dependent scaling of Crus I in ASD suggests lateral cerebellar circuits do not adapt sensory prediction and error processes to maintain precision motor output during more demanding conditions. Together, our results demonstrate multiple cortical-cerebellar mechanisms associated with sensorimotor imprecision in ASD.

3195

\section{Genomic Analysis of Primary Plasma Cell Leukemia reveals Complex Cytogenetic Alterations and High Risk Mutational Patterns}

Carolina Schinke ${ }^{1}$, Eileen Boyle, Cody Ashby, Yan Wang, Davies, Christopher Wardell, Sharmilan Thanendrarajan, maurizio Zangari, Frits van Rhee, Gareth Morgan and Brian Walker ${ }^{1}$ University of Arkansas Translational Research Institute

OBJECTIVES/SPECIFIC AIMS: 1) Determine the mutational landscape, including translocation, mutations and mutational signatures as well as copy number variations of pPCL and identify significant 
differences to non pPCL MM. 2) Determine whether genetic changes pertinent to PPCL could be explored as therapeutic targets to improve the dismal prognosis of this patient population. METHODS/STUDY POPULATION: Samples from overall $19 \mathrm{pPCL}$ patients that presented to the Myeloma Center, UAMS between 2000-2018 were used for this study. We performed gene expression profiling (GEP; Affymetrix U133 Plus 2.0) of matched circulating peripheral PCs and bone marrow (BM) PCs from 13 patients. Whole exome sequencing (WES) was performed on purified CD138+ PCs from BM aspirates from 19 pPCL patients with a median depth of $61 \mathrm{x}$. CD34+ sorted cells, taken at the time of stem cell harvest from the same 19 patients, were used as controls. Translocations and mutations were called using Manta and Strelka and annotated as previously reported. Copy number was determined by Sequenza. RESULTS/ANTICIPATED RESULTS: 1) GEP from the BM and circulating peripheral PCs showed that the expression patterns of the two samples from each individual clustered together, indicating that circulating PCs and BM PCs in PPCL result from the same clone and are biologically clearly related. 2) The clinical characteristics from the patient cohort used for WES analysis were as follows: median age was 58 years (range 36-77), females accounted for 74\% (14/19), an elevated creatinine level was found in 78\% (14/18) and an elevated LDH level in 71\% $(10 / 14)$. All patients presented with an ISS stage of III. Median OS of the whole dataset was poor at 22 months, which is consistent with OS from previously reported pPCL cohorts. 3) Primary Immunoglobulin translocations were common and identified in $63 \%(12 / 19)$ of patients, including MAF translocations, which are known to carry high risk in $42 \%(8 / 19)$ of patients $[\mathrm{t}(14 ; 16)$, $32 \%$ and $\mathrm{t}(14 ; 20), 10 \%]$ followed by $\mathrm{t}(11 ; 14)(16 \%)$ and $\mathrm{t}(4 ; 14)$ (10\%). Furthermore, 32\% (6/19) of patients had at least one MYC translocation, which are known to play a crucial role in disease progression. 4) The mutational burden of PPCL consisted of a median of 98 non-silent mutations per sample, suggesting that the mutational landscape of PPCL is highly complex and harbors more coding mutations than non-pPCL MM. 5) Driver mutations, that previously have been described in non-pPCL MM showed a different prevalence and distribution in PPCL, including KRAS and TP53 with 47\% (9/19) and 37\% (7/19) affected patients respectively compared to $21 \%$ and $5 \%$ in non-PCL MM. PIK3CA (5\%), PRDM1 (10\%), EP300 (10\%) and NF1 (10\%) were also enriched in the PPCL group compared to previously reported cases in nonpPCL MM. 6) Biallelic inactivation of TP53 - a feature of Double Hit myeloma - was found in 6/19 (32\%) samples, indicating a predominance of high risk genomic features compared to nonpPCL MM. Furthermore, analysis of mutational signatures in pPCL showed that aberrant APOBEC activity was highly prevalent only in patients with a MAF translocation, but not in other translocation groups. DISCUSSION/SIGNIFICANCE OF IMPACT: In conclusion we present one of the first WES datasets on PPCL with the largest patient cohort reported to date and show that PPCL is a highly complex disease. The aggressive disease behavior can, at least in part, be explained by a high prevalence of MAF and MYC translocations, TP53 and KRAS mutations as well as bi-allelic inactivation of TP53. It is of interest that only KRAS but not NRAS mutations are highly enriched in PPCL. From all highly prevalent genomic alterations in PPCL, only KRAS mutations offer a potential for already available therapeutically targeting with MEK inhibitors, which should be further explored.
Hemoglobin selectively inhibits renal proximal tubular uptake of proteins: Implications for vitamin D deficiency and kidney disease in sickle cell disease

Megan L. Gliozzi ${ }^{1}$, Youssef Rbaibi, Dario A. Vitturi, Jesús Tejero and Ora A. Weisz

${ }^{1}$ University of Pittsburgh

OBJECTIVES/SPECIFIC AIMS: While Hb-induced toxicity has been assumed to cause PT dysfunction, the development of tubular proteinuria from this dysfunction in SCD patients is not well understood. We previously found that free $\mathrm{Hb}$, at concentrations predicted to be present chronically and during hemolytic crisis in the tubular filtrate of SCD patients, impairs uptake of albumin by PT cells via direct competition for binding to megalin and cubilin receptors. The purpose of this study is to further evaluate the consequences of increased filtered $\mathrm{Hb}$ concentrations on vitamin $\mathrm{D}$ reabsorption and activation. METHODS/STUDY POPULATION: We have developed a PT cell culture model that closely mimics in vivo PT cell structure, morphology, and endocytic capacity. Using this model, we treated cells with physiologic levels of cell-free $\mathrm{Hb}$ estimated in SCD and measured protein endocytosis and toxicity/oxidative stress. Endocytosis of fluorescently-tagged DBP and RBP were evaluated and quantified by confocal imaging and spectrofluorometry. Cellular toxicity and oxidative stress were assessed by measuring aconitase activity and accumulation of mitochondrial reactive oxygen species. RESULTS/ANTICIPATED RESULTS: PT cell uptake of DBP was significantly inhibited by both concentrations of Hb estimated to be filtered into the tubule lumen under chronic conditions $(0.6 \mu \mathrm{M} \mathrm{Hb} ; 39 \%$ inhibition) and hemolytic crisis ( $\leq 20 \mu \mathrm{M} \mathrm{Hb}$; up to $92 \%$ inhibition) in SCD patients. ${ }^{* * *} \mathrm{p}<0.0001$ by one-way ANOVA, Dunnett's multiple comparisons test. PT cell uptake of RBP was minimally affected by the same concentrations of $\mathrm{Hb}$ that profoundly inhibited internalization of DBP. RBP uptake was not significantly inhibited by all concentrations of $\mathrm{Hb}$ tested except the estimated hemolytic crisis maximum concentration $(20 \mu \mathrm{M} ; 27 \%$ inhibition). RBP uptake inhibition at $20 \mu \mathrm{M} \mathrm{Hb}$ treatment was dramatically less than DBP uptake inhibition under the same treatment condition (27\% RBP inhibition vs $92 \%$ DBP inhibition). ${ }^{*} \mathrm{p}<0.05$ by oneway ANOVA, Dunnett's multiple comparisons test. Mitochondrial oxidative stress, measured as a decrease in aconitase activity, was significantly increased in cells exposed to $\mathrm{Hb}(\sim 43 \%$ aconitase activity reduction after $72 \mathrm{~h} 20 \mu \mathrm{M} \mathrm{Hb}$ treatment, and $\sim 11 \%$ aconitase activity reduction after $72 \mathrm{~h} 1 \mu \mathrm{M} \mathrm{Hb}$ treatment). ${ }^{*} \mathrm{p}<0.05,{ }^{* *} \mathrm{p}<0.01$ by onesample t-test of the differences between treatment and untreated control conditions. We are currently assessing changes in PT cell vitamin D hydroxylase expression levels and vitamin D metabolism after exposure to chronic and hemolytic concentrations of $\mathrm{Hb}$. Because oxidative stress has been previously reported to affect hydroxylase expression and activity, we expect to find a decrease in vitamin $\mathrm{D}$ hydroxylase expression and/or activity, resulting in decreased vitamin D activation. DISCUSSION/SIGNIFICANCE OF IMPACT: Our results suggest that competition for megalin/cubilin binding between $\mathrm{Hb}$ and normally-filtered proteins, including DBP, may be the primary cause of tubular proteinuria in SCD patients. This inhibition appears to be selective for proteins that are largely $\alpha$-helical in structure, such as albumin and DBP. Understanding the structural basis for $\mathrm{Hb}$ competition with filtered proteins for PT uptake could identify biomarkers to detect tubular proteinuria in SCD patients prior to the onset of kidney disease. This may also help develop therapeutic compounds that would selectively inhibit 